Revue de droit comparé du travail et de la sécurité sociale

$3 \mid 2020$

La Directive 2019/1158 du 20 juin 2019 concernant l'équilibre entre vie personnelle et vie privée des parents et des aidants

\title{
Le monde du travail face au Covid-19 en Turquie
}

Melda Sur

\section{OpenEdition}

Journals

Édition électronique

URL : https://journals.openedition.org/rdctss/1042

DOI : $10.4000 /$ rdctss. 1042

ISSN : 2262-9815

Éditeur

Centre de droit comparé du travail et de la sécurité sociale

Édition imprimée

Date de publication : 1 novembre 2020

Pagination : 226-229

ISSN : $2117-4350$

Référence électronique

Melda Sur, "Le monde du travail face au Covid-19 en Turquie », Revue de droit comparé du travail et de la sécurité sociale [En ligne], 3 | 2020, mis en ligne le 01 novembre 2021, consulté le 11 novembre 2021. URL : http://journals.openedition.org/rdctss/1042 ; DOI : https://doi.org/10.4000/rdctss.1042

\section{c) (i) (9)}

Revue de droit comparé du travail et de la sécurité sociale est mise à disposition selon les termes de la Licence Creative Commons Attribution - Pas d'Utilisation Commerciale - Pas de Modification 4.0 International. 


\section{LE MONDE DU TRAVAIL FACE AU COVID-19 EN TURQUIE}

Après avoir effectué un bref focus sur un récent arrêt de la Cour constitutionnelle confirmant sa jurisprudence sur la protection du droit de grève (I), il sera question de la protection de l'emploi face à la pandémie du Covid-19, (II) et de l'usage de plus en plus répandu du travail à distance (III).

La problématique réside notamment dans l'équilibre à établir entre la sauvegarde de la santé et de la sécurité des travailleurs, tout en permettant aux entreprises et aux services publics de poursuivre leurs activités : est-il possible de sauvegarder l'emploi et d'assurer un revenu décent à tous les travailleurs, tout en protégeant les entreprises mises en difficulté face à la pandémie, et ainsi de maintenir l'économie nationale toute entière?

\section{I - LA COUR CONSTITUTIONNELLE GARANTE DU DROIT DE GRÈVE}

Par un arrêt du 14 novembre 20191', La Cour Constitutionnelle annule la disposition de l'article 63 qui avait élargi les pouvoirs présidentiels et permettait d'ajourner des grèves dans les banques et les transports publics urbains². Publié en février 2020, cet arrêt se réfère en particulier aux conclusions des organes de contrôle de I'OIT pour lesquels ces services ne sauraient être considérés comme essentiels ${ }^{3}$. Le juge constitutionnel maintient sa jurisprudence et demeure gardien des droits fondamentaux garantis par la Constitution turque et la Convention européenne des droits de l'Homme, en adoptant une conception relativement large intégrant la jurisprudence de la Cour européenne, l'interprétation des organes de contrôle de l'OIT et de la Charte Sociale Européenne.

Cet arrêt semble conclure une série d'épisodes concernant l'interdiction légale depuis 2012 des grèves dans les banques et les transports publics urbains; interdiction qui fut annulée une première fois par la Cour Constitutionnelle ${ }^{4}$. En réaction à cette première décision, le gouvernement obtient la faculté de suspendre ces mêmes grèves ${ }^{5}$. La Cour Constitutionnelle, conformément à sa jurisprudence antérieure, annula cette fois-ci ces cas de suspension des grèves les considérant contraires aux droits syndicaux garantis par la Constitution (art. 51 et 54).

1 Arrêt n²018/90 - 2019-85, publié au Journal Officiel du 13 février 2020, n³1038.

2 Pour l'historique, voir la rubrique des Actualités juridiques internationales «Turquie », Revue de droit comparé du travail et de la sécurité sociale, 2017/1, p. 208.

3 La Cour fait référence à l'exposé des motifs de son précédent arrêt du 22.10.2014 (n²013/12014/161) sur l'inconstitutionnalité de l'interdiction des grèves dans ces secteurs.

4 Arrêt du 22 octobre 2014, n² 2013/1-2014/161, Journal Officiel du 11 novembre 2015, n²9529. Voir Actualités juridiques internationales "Turquie ", Revue de droit comparé du travail et de la sécurité sociale, 2017/1, op.cit.

5 Ordonnance-Loi du 31 octobre 2016 n³5, puis loi du 1er février 2018 n 7071 modifiant l'article 63 de la Loi $n^{\circ} 6356$ sur les Syndicats et les conventions collectives de travail. 


\section{II - LA PROTECTION DE L'EMPLOI FACE AU COVID-19}

La vie économique et le monde du travail sont fortement éprouvés par l'épidémie Covid-19. La Turquie, touchée par cette crise, a eu recours à des mesures visant à protéger l'emploi et à assurer un certain revenu aux salariés se trouvant au chômage. Ainsi, pour protéger les salariés contre les pertes d'emploi, l'État a pris trois types de mesures : faciliter le recours au chômage partiel (A), aux congés sans solde assortis d'un revenu minimum garanti (B), et restreindre la faculté de licencier (C).

\section{A - LE RECOURS AU TRAVAIL RÉDUIT}

Le régime du " travail réduit " (kısa çalışma) en Turquie correspond, avec quelques différences, au chômage partiel en droit français. Ce régime est applicable dans les cas de crise économique générale, sectorielle ou régionale, et en cas de force majeure causant temporairement une importante réduction (d'un tiers au moins) des heures de travail, ou une cessation ou baisse d'activité s'étendant à quatre semaines ou plus. Les épidémies comptent parmi les cas de force majeure ${ }^{6}$. La loi requiert certaines conditions d'admissibilité, et exige une démarche de l'employeur auprès des autorités du Ministère du travail, pour que puissent être indemnisées les heures et les journées non travaillées. La demande doit être dûment motivée et documentée, adressée aux services départementaux du Ministère du travail (Institution de l'Emploi - Türkiye İş Kurumu - Işkur). Cette requête sera évaluée par les services d'inspection du travail.

Suivant des modifications apportées à la Loi n²447 sur l'Assurance-chômage, cette procédure a été assouplie et les conditions allégées à l'occasion de la pandémie ${ }^{7}$. Ainsi, les demandes sont prises en compte dès leur soumission et l'indemnisation immédiatement versée (article provisoire 25). S'il s'avère par la suite que les conditions n'étaient pas remplies, alors l'indemnité doit être restituée et une amende administrative payée. Pour avoir droit à l'allocation de travail réduit - à la place des 600 jours travaillés et cotisés normalement nécessaires pour bénéficier de l'allocation-chômage - il suffira d'avoir cotisé 450 jours sur les 3 dernières années (Loi sur l'Assurance-chômage, art. provisoire 23). Si la demande aboutit, le salarié a ainsi droit à «l'allocation de travail réduit » (kısa çalışma ödeneği) versée chaque mois par les services de l'Emploi (İşkur). Le montant fixé à $60 \%$ du salaire brut moyen des 12 derniers mois, est plafonné à $150 \%$ du salaire minimum brut ${ }^{8}$. En cas de reprise normale du travail, l'État subventionnera les entreprises en versant pendant 3 mois toutes les primes d'assurance, en s'appuyant sur le fonds de l'assurance-chômage (Loi sur l'Assurance-chômage, art. provisoire 26). La durée de cette couverture qui est d'ordinaire de 3 mois, peut être étendue jusqu'au 31 décembre 2020.

6 Règlement sur le travail réduit et son indemnisation (Kısa Çalışma ve Kısa Çalışma Ödeneği Hakkında Yönetmelik), art. 3. Le Code du travail prévoit par ailleurs dans les cas de force majeure, que la moitié du salaire soit versée par l'employeur durant une semaine (Code du travail, art. 40). Aussi une fois la période d'une semaine passée, ce sera aux services de l'Emploi de verser l'allocation de travail réduit et de cotiser pour l'assurance-santé.

7 Loi $n^{\circ} 7226$ publiée au Journal Officiel du 26 mars 2020, $n^{\circ} 31080$ bis ; Loi $n^{\circ} 7244$ publiée ou Journal Officiel du 17 avril 2020, n³1102 et Loi n7252 publiée au Journal Officiel du 28 juillet 2020, n³1199.

8 Art. Additionnel 2 de la Loi n 4447 sur l'Assurance-chômage (İşsizlik Sigortası Kanunu). Le salaire minimum net mensuel est actuellement de 2324,70 livres turques (261€). 


\section{B - LE RECOURS UNILATÉRAL AU CONGÉS SANS SOLDE}

Constituant une modification essentielle du contrat de travail et devant être normalement effectuée par accord mutuel, la mise en congé sans solde (ücretsiz izin) est désormais devenue une faculté reconnue à l'employeur pour une durée allant jusqu'à 3 mois. Bien qu'étant une modification essentielle du contrat en défaveur du salarié, elle ne constituera désormais plus pour celui-ci une cause justifiant la rupture du contrat ${ }^{9}$.

En contrepartie, le travailleur se verra allouer par l'État un montant journalier de 39.24 livres turques, soit 1177 livres turques par mois ${ }^{10}$. La modicité de la somme est évidente. Cette allocation est prise sur le fonds de l'assurance-chômage ; y auront droit ceux qui n'ont pas réuni les conditions requises pour bénéficier de l'indemnité de travail réduit ou de l'assurance chômage; il s'agit donc d'un pis-aller. Les personnes concernées, ainsi que leur famille, sont néanmoins couvertes par l'assurance-maladie dont les cotisations seront prises en charge sur le fonds de l'assurance-chômage (article provisoire 24) ${ }^{11}$.

\section{C - LES RESTRICTIONS AUX LICENCIEMENTS}

Allant de pair avec la faculté de mettre le salarié en congé sans solde, la loi interdit le licenciement, hormis en cas de situations à peu près équivalentes à celles de la faute lourde en droit français. La liste - non exhaustive - des principales causes est énumérée à l'article 25/II du Code du Travail. Mais la législation du travail, et notamment le Code des obligations, permet de licencier dans des cas où l'employeur ne saurait être raisonnablement tenu de poursuivre la relation de travail. Suite à une révision récente, la loi accorde la possibilité de mettre fin au contrat de manière plus large, en incluant l'arrivée à terme de contrats à durée déterminée, la fermeture et la cessation d'activité de l'établissement, la fin de la sous-traitance et l'achèvement des travaux de construction qui pourront également justifier les ruptures ${ }^{12}$. L'application de ces dispositions peut être prolongée par décret présidentiel pour des durées successives de 3 mois, et ce jusqu'au 30 juin 2021, une date assez éloignée qui présage des effets de la pandémie sur une durée longue.

\section{III - VERS UNE NOUVELLE CONCEPTION DES RELATIONS SALARIALES ?}

Durant le mois d'août dernier, le Ministère de la Santé a constaté une nette augmentation des cas de Covid-19. Depuis le début de la pandémie (et au moment où nous rédigeons ces lignes), la Turquie comptabilise ainsi plus de 6000 décès, et plus de 700 malades se trouvant dans un état grave. Devant un tel tableau, les restrictions impactant les activités économiques, commerciales, ainsi - et surtout - que celles liées aux domaines de l'éducation et de la culture, vont se prolonger. Les secteurs les plus touchés sont le tourisme, l'hôtellerie et la restauration, et tout le domaine de la culture, arts et spectacles qu'il semble donc nécessaire de soutenir plus particulièrement.

Le taux de chômage officiellement annoncé en avril 2020 était de 12,8\% (3.775.000 personnes). On dénombre, toujours d'après ces statistiques, $28,7 \%$ de travail non déclaré.

9 Article provisoire 10 du Code du Travail, inséré par la Loi nº7244 du 16 avril 2020.

10 Soit environ $4,40 €$ par jour et $132 €$ par mois.

11 La durée de cette aide, qui peut être prolongée jusqu'à 6 mois par décret présidentiel, ne peut dépasser cependant celle de l'indemnisation du chômage partiel.

12 Loi n $^{\circ} 252$, Journal Officiel du 28 juillet 2020, n³1199 et article provisoire 10 du Code du Travail. 
Toutefois, le chômage non déclaré est lui-même considérable et les milieux syndicaux et académiques ont fait remarquer que ces chiffres ne prenaient pas en considération tous ceux qui, ayant perdu l'espoir d'être embauchés, ne s'adressent plus à l'agence pour l'emploi (qui prend en compte seulement ceux inscrits les 4 dernières semaines), ni le nombre considérable de salariés mis en congé sans solde et en chômage partiel.

La faculté de résilience de l'économie turque et de la population, qui demeure la plus jeune d'Europe (13 millions de personnes de $25-24$ ans, soit $15,6 \%$ de la population $)^{13}$, combinée à une forte solidarité familiale et sociale, ont permis jusqu'à présent une certaine maîtrise de la situation économique et sociale. Cependant, le problème se révèle de plus en plus grave, en particulier dans le domaine de l'enseignement public et des universités où l'enseignement se fait à distance, avec des résultats que l'on imagine.

Le travail à distance, sous sa forme la plus répandue, est actuellement le télétravail (souvent un travail en ligne), réglementé de manière assez succincte en droit turc par l'article 14 du Code du travail. Ces dispositions exigent un contrat écrit dans lequel seront indiqués la définition du poste de travail, le mode d'exécution, la durée et le lieu de travail, le salaire et son versement, l'équipement fourni par l'entreprise et sa protection, le mode de communication et les conditions générales et spéciales du travail (art. 25/5). L'équipement (ordinateur, etc.) sera fourni par l'entreprise, et les frais et dépenses encourus remboursés par l'employeur (Code des Obligations, arts. 414 et 416).

Une difficulté de taille consiste à délimiter et à définir la durée du temps de travail : souvent le salarié reste à la disposition de l'employeur pendant un temps assez prolongé et, dans certains secteurs, il est tenu d'exécuter le travail demandé à toute heure. Les limitations légales sur la durée de travail et les droits aux congés payés qui doivent pourtant être respectés, demeurent parfois lettre morte. La loi interdit d'exercer toute discrimination, sauf raison essentielle justifiant la différence de traitement (art. 14/6); par exemple, le repas servi sur le lieu de travail ne sera pas exigible, toutefois un versement compensatoire serait adéquat ; inversement, les frais de transport ne seraient pas dus dans les cas de travail à domicile.

Une autre difficulté à trait à la santé et la sécurité du travail : comment assurer le respect des mesures nécessaires à la santé et la sécurité du travail sans exercer une intrusion au domicile du salarié ? On estime que la responsabilité d'instruire et d'informer le travailleur s'impose de préférence à l'exercice d'un contrôle sur place (art. 14/6) ${ }^{14}$. Comme une réglementation trop détaillée ne semble pas être de mise, le principe de bonne foi associé à celui de précaution permettront de résoudre nombre des problèmes évoqués ci-dessus.

En conclusion, l'épidémie Covid-19 a bouleversé la vie individuelle et collective et a donné lieu à une douloureuse remise en question des certitudes et habitudes qui semblaient jusqu'à présent bien acquises. Le monde du travail en a durement souffert, et le recours accru aux technologies informatiques semble désormais un phénomène irréversible dans bien des secteurs. Reste à rechercher la formule qui permettra de sauvegarder en même temps l'emploi, la santé, la sécurité des travailleurs et de la population entière, tout en gardant une économie et des services publics performants.

13 Statistiques du TUiK (Türkiye İstatistik Kurumu) publiées le 15 mai 2020.

14 Voir à ce sujet M. Alp, Tele Çalışma (Uzaktan Çalışma), Sarper Süzek'e Armağan, I, İstanbul, 2011, p. 795 ; M. Kandemir, Iş Hukuku ve Sosyal Güvenlik Hukuku Açısından Tele Çalışma, İstanbul, 2011, p. 118 ; N. Çelik, N. Caniklioğlu, T. Canbolat, İ̧ Hukuku Dersleri, 32. éd., İstanbul, 2019, p. 216. 